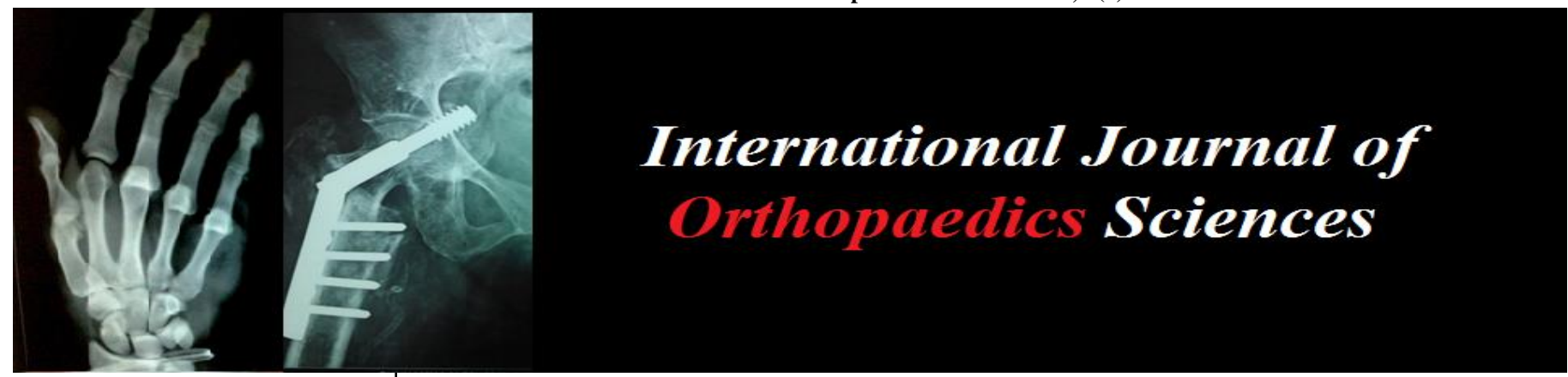

ISSN: $2395-1958$

IJOS 2019; 5(3): 94-96

(C) 2019 IJOS

www.orthopaper.com

Received: 27-05-2019

Accepted: 30-06-2019

Mohammed Jalal Mohiuddin

Assistant Professor, Department of Orthopedics, Deccan College of Medical Sciences, Hyderabad, Telangana, India

Syed Farooq Iftekhar Ahmed Medical Student, Deccan College of Medical Sciences Hyderabad, Telangana, India

\section{Jawad Mirza}

Medical Student, Deccan College of Medical Sciences, Hyderabad,

Telangana, India

\section{Abdul Hai Mohammed}

Medical Student, Deccan College of Medical Sciences Hyderabad,

Telangana, India

\section{Sham sunder}

Professor and Head, Department of Orthopedics, DCMS,

Hyderabad, Telangana, India

\section{Musculoskeletal tuberculosis: A diagnostic approach}

\section{Mohammed Jalal Mohiuddin, Syed Farooq Iftekhar Ahmed, Jawad Mirza, Abdul Hai Mohammed and C Sham sunder}

DOI: https://doi.org/10.22271/ortho.2019.v5.i3b.1515

\section{Abstract}

Musculoskeletal tuberculosis constitutes 3 percent of extra pulmonary secondary tuberculosis predominantly involving the spine. The variations in its presentation and unclear symptoms make diagnosis difficult in early period. The normal lab values like ESR, TLC, DLC and no clear history of previous pulmonary infection makes the picture unclear and delays the diagnosis.

Present work is the overview of the presentation of various musculoskeletal presentations, efficacy of BCG vaccination, time delay in diagnosis, laboratory parameters, $x$ rays and other radiological changes etc. The reliability of the various lab procedures like AFB stain, histopathology, tuberculosis bacteria culture and cartridge-based nucleic acid amplification test (CBNAAT) has also been overviewed.

Keywords: Musculoskeletal tuberculosis, extra pulmonary, spine, CBNAAT

\section{Introduction}

Tuberculosis (TB) remains the most common cause of death from infectious disease worldwide (1).In India every year almost twelve lakh patients are notified as having newly diagnosed TB (2).85 percent of them with pulmonary tuberculosis and 15 percent extra pulmonary lesions of which 1 to 3 percent are bone and joint lesions (3).

Bone and Joint tuberculosis results from haematogenous spread from a pulmonary or other visceral or lymph node focus (4). Direct manifestations of TB musculoskeletal involvement include spondylitis, septic arthritis, osteomyelitis, myositis, bursitis, subcutaneous abscesses, or tenosynovitis. Immunologic reactions such as reactive arthritis (Poncet's), erythema nodosum, erythema induratum or secondary amyloidosis may also manifest as tubercular rheumatism (5). Tuberculous osteomyelitis is an uncommon form of extra-pulmonary tuberculosis, representing less than five percent of cases of osteoarticular tuberculosis (6).

Variation in presentation and atypical symptoms delay the diagnosis many times. The duration of symptoms at diagnosis ranges from 2 weeks to several years. Back pain is usually present (83-100\%) in potts spine, but only one-third of patients have fever or constitutional symptoms. Hematological and clinical data contribute little to the diagnosis, and leukocyte count is usually normal. The erythrocyte sedimentation rate (ESR) and C-reactive protein concentration are often raised, but levels are lower than those seen in pyogenic vertebral infections. The tuberculin skin test is usually positive, although it is non-diagnostic in endemic regions and may be negative in immune deficient patients (7). Average age group affected in musculoskeletal tuberculosis is 23.6 years (8). The concurrent pulmonary tuberculosis in the patients varies $19 \%$ to $42.6 \%$ with marginal female predominance (9).Biopsy supposed to be the gold standard test for diagnosis has confirmation rate of possible TB pathological findings in patients with extra pulmonary TB in $80.4 \%$ patients (10). Protective efficacy of BCG remains controversial, varying from $0 \%$ to $80 \%$ in different populations and geographic regions (11).Acid-fast bacillus (AFB) stain and AFB culture tests are positive in fewer than $10 \%$ and $20 \%$ of suspected cases (12). Approximately 50 percent of patients with bone and joint tuberculosis have negative findings on chest X-ray (13). About $70 \%$ of musculoskeletal tuberculosis has positive $\mathrm{x}$ ray findings (14).MRI has $88.2 \%$ specificity and very high sensitivity for the infection (15).

This study is to focus on the demographic distribution, variations in diagnostic (lab and radiology) parameters and various involvements of musculoskeletal system in tuberculosis.

\section{Correspondence
Mohammed Jalal Mohiuddin} Assistant Professor, Department of Orthopedics, Deccan College of Medical Sciences, Hyderabad, Telangana, India 


\section{Aims and Objectives}

- Analyze various clinical presentation of musculoskeletal tuberculosis.

- Evaluate the role of various diagnostic parameters.

- Comparison with previous studies published in literature.

\section{Methods \\ Description of study}

IRB clearance has been obtained prior to the study. It's an observational study in which all cases of osteoarticular tuberculosis diagnosed in Deccan College of Medical Sciences, analyzed for various epidemiologic parameters like age, sex, anatomical site, and delay in presentation, associated pulmonary TB, blood and radiological markers. The study Period extended from September 2017 to September 2018, with sample size of 25 cases.

\section{Inclusion criteria}

All cases of musculo skeletal tuberculosis diagnosed in the institution and the cases diagnosed outside and referred to the institute are also included in study.

\section{Collection of Data}

The relevant History and the clinical examination, documented. Cases with clinical suspicion of tuberculosis lesion are subjected for the $\mathrm{x}$-rays and blood investigation. Tissue collected for diagnosis in easily approachable regions and MRI for deep lesion (like spine).

\section{Results}

Total number of patients included is 25 (9 males and 16 females).Age of the patients ranges from 13 years to 65 years with mean age of 30.1 years. BMI of patients ranged from 18.75 to 34.63 with mean BMI of 30.1.Most of the patient belongs to lower- upper class. 2 patients $(10 \%)$ have history of primary pulmonary tuberculosis and $25 \%$ (5 patients) has history of contact tuberculosis. BCG administration, confirmed by the BCG scar seen in 17 patients(85\%). Three patients $(15 \%)$ has co morbidities include diabetes and chronic kidney disease, one had rheumatoid arthritis and on methotrexate.

Duration of symptoms were average of 5.05 months(maximum of 24 months and minimum of 3 weeks).No of visits to the same clinician before diagnosis ranges between 2 to 4 and number of clinician changed before diagnosis were three.

15 patients had normal ESR $(<22)$ and 17 patients has normal TLC (total leucocytes count) and DLC (differential leucocytes count) counts.

14 patients under went biopsy and it came positive showing caseation necrosis and granuloma. AFB stain done for 14 patients which reported positive for two samples and culture positive in 11 patients. CBNAAT sample reported positive in 11 out of 14 patients and one out of it had rifampicin resistance.

Distribution according to site involved is spine in 13 patients, dactylitis in 2 patients.

Two patients involving knee and wrist joint each. One patient each involving hip joint, sacroiliac joint, multifocal lesion, facial bones, ankle joint and tibia.

\section{Discussion}

Musculoskeletal tuberculosis is one of the difficult presentations which resembles other pathologies and make diagnosis difficult. It mostly affects female that is around 64 percent and involves spine in 52 percent of patients. The atypical clinical presentation and normal basic blood parameters makes delay in the diagnosis.

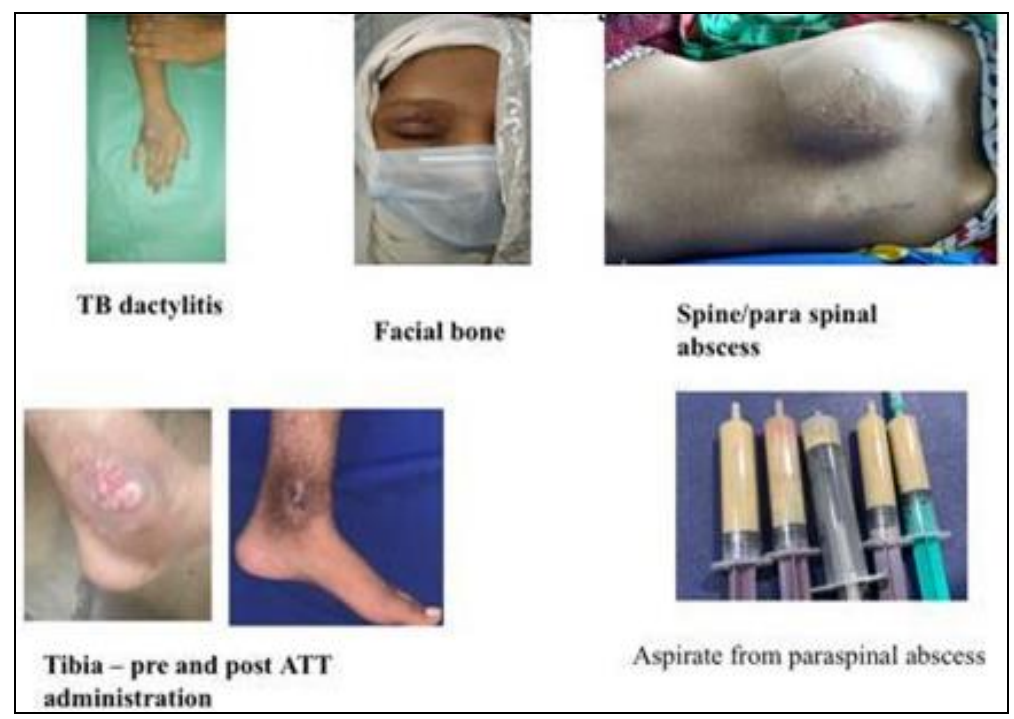

Fig 1: Clinical photograph

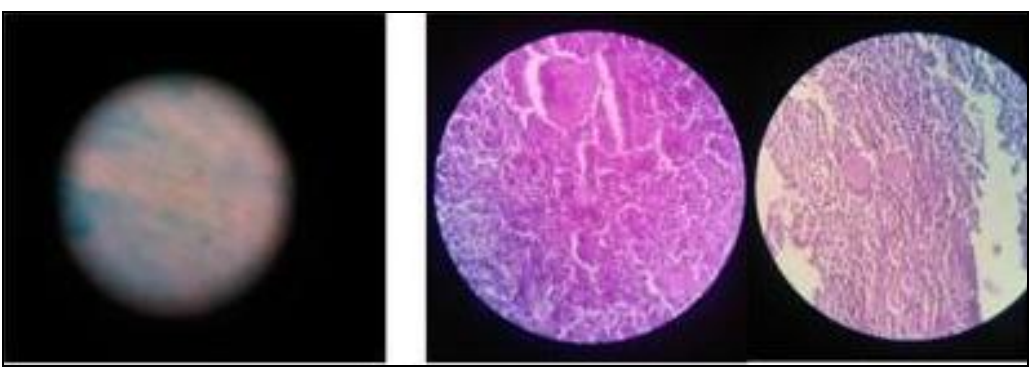

Fig 2: (A) Microscopy/AFB stain (B) Histo pathology- granuloma/caseation necrosis $\sim 95 \sim$ 


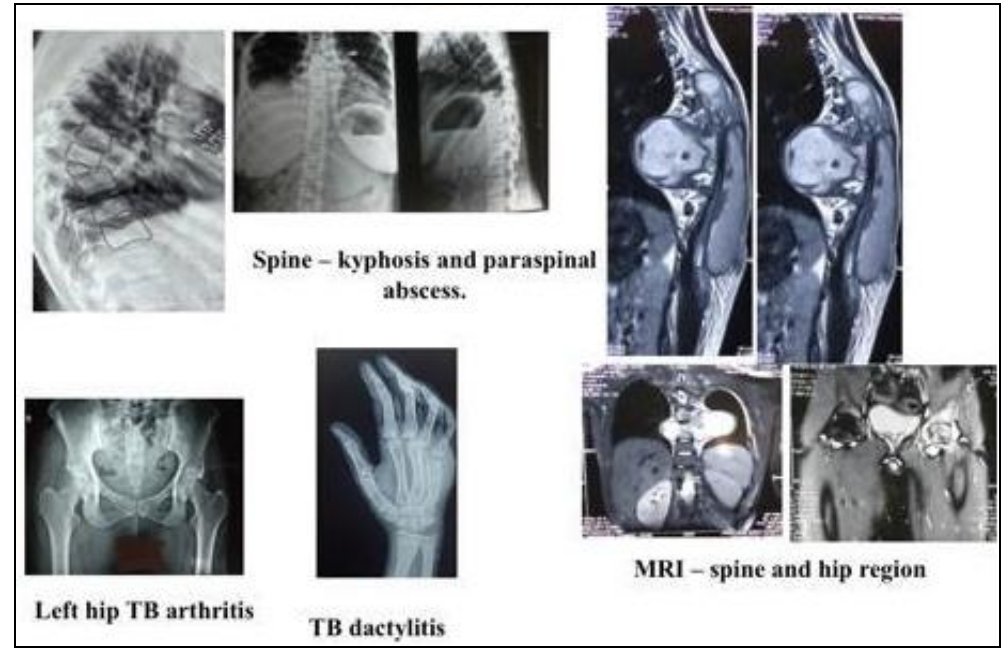

Fig 3: Radiology/imaging

\section{Conclusion}

Secondary tuberculosis is the upcoming challenge in modern era and development of resistance to primary antitubercular drugs adding to it. Early diagnosis needs strong suspicion and thorough examination. Orthopedic surgeon must have orientation to various possible atypical presentations.

By early diagnosis the disease can be treated earliest without much morbidity and residual sequelae.

\section{References}

1. World Health Organization. Stop TB annual report 2001. Geneva: WHO, 2002.

2. Wares F, Balasubramanian R, Mohan A, Sharma SK. Extra pulmonary Tuberculosis: Management \& Control. In Agarwal and Chauhan, In Tuberculosis Control in India. Directorate General of Health Services/Ministry of Health and Family Welfare. Elsevier: India, 2005, 95114.

3. Haider ALM. Bones and Joints Tuberculosis. Bahrain Medical Bulletin. 2007; 29:1-9.

4. Enache SD, Pleasea IE, Anusca D, Zaharia B, Pop OT. Osteoarticular tuberculosis-a ten years case review Rom. J Morphol Embryol. 2005; 46:67-72.

5. Gupta N, Bhatnagar AK. Musculoskeletal manifestations of tuberculosis: An observational study. J Family Med Prim Care. 2018; 7(3):538-541.

6. Rieder HL, Snider DE Jr., Cauthen GM. Extrapulmonary tuberculosis in the United States. American Review of Respiratory Disease. 1990; 141(2 I):347-351.

7. Pigrau-Serrallach C, Rodríguez-Pardo D. Bone and joint tuberculosis. European spine journal, 22(1 4), 556-566.

8. Ahmad NAF, Huda N. Osteoarticular Tuberculosis-A Three Years Retrospective Study. Journal of Clinical and Diagnostic Research. 2013; 7(10):2189-2192.

9. Shiva Prasad1, Anupam Wakhlu, et al., Features of Extra-spinal Musculoskeletal Tuberculosis: A Retrospective Study from a North Indian Tertiary Care Institute, Indian journal of rheumatology. 2017; 2(3):146151.

10. Kyung Min Bae, Sung-Chul Lim. The Relevance of Biopsy in Tuberculosis Patients Without Human Immunodeficiency Virus Infection, Am J Trop Med Hyg. 2015; 92(3):636-640.

11. Fine PE. Variation in protection by BCG: implications of and for heterologous immunity. Lancet. 1995; 3(6):13391345.

12. Chakravorty S, Sen MK, Tyagi JS. Diagnosis of extrapulmonary tuberculosis by smear, culture, and using universal sample processing technology. J Clin Microbiol. 2005; 43:4357-4362.

13. Davidson PT, Horowity I. Skeletal tuberculosis: A review with patient presentations and discussions. Am J Med. 1970; 48:77-84.

14. Rivas-Garcia A, Sarria-Estrada S, Torrents-Odin C, Casas-Gomila L, Franquet E. Imaging findings of Pott's disease. European Spine Journal. 2013; 22(4):567-578.

15. Jain AK, Sreenivasan R, Saini NS, Kumar S, Jain S, Dhammi IK. Magnetic resonance evaluation of tubercular lesion in spine. Int Orthop. 2012; 36:261-269. 